International Journal of Neural Systems, Vol. 7, No. 2 (May, 1996) 213-218

(C) World Scientific Publishing Company

\title{
Corrigendum
}

James J. Knierim, Hemant S. Kudrimoti

and Bruce L. McNaughton

\section{NEURONAL MECHANISMS UNDERLYING THE INTERACTION BETWEEN VISUAL LANDMARKS AND PATH INTEGRATION IN THE RAT}

\author{
[Reprinted from International Journal of Neural Systems, \\ Supplementary Issue, 1995, 95-100]
}

\begin{abstract}
Place- and direction-specific firing properties of hippocampal and thalamic neurons are not strongly tied to visual landmarks when a rat is disoriented. These results suggest that rats rely more on path integration mechanisms than on landmarks, until they have learned that the landmarks are stable directional references.
\end{abstract}

Imagine getting off a bus at a stop where you have visited only once or twice before. As you get off the bus, you are uncertain of the direction you need to head to make your appointment, and you begin to wander, feeling disoriented. After a minute, you recognize a bank on the corner, and suddenly everything snaps into place; you realize you are heading in the wrong direction, so you turn around and proceed directly to your destination. Somehow, the sight of the familiar landmark has oriented your "cognitive map" of the area, and you are then able to navigate efficiently to your goal. The neuronal mechanisms underlying the internal representation of place and direction, and the ability to use this information to navigate, are the subjects of this paper.

The one brain structure most often identified with spatial learning and navigation is the hippocampus. In 1978, O'Keefe and $\mathrm{Nadel}^{1}$ proposed that the hippocampus formed a cognitive map of the environment, whereby animals and humans learn to navigate in familiar and unfamiliar environments. This theory was based on two major lines of evidence:

1. Behavioral studies demonstrated that hippocampal lesions almost always cause an impairment in spatial navigation tasks, whereas other types of learning abilities are unaffected., ${ }^{1,2}$
2. Neurophysiological studies demonstrated that the most striking correlate of hippocampal neuronal firing is the spatial location of the rat. $^{3}$ Although many investigators believe that the hippocampus plays a more general role in learning than just spatial learning, ${ }^{4,5}$ it is generally agreed that a major, if not exclusive, function of the hippocampus and related structures is spatial learning.

The so-called place cells of the rat hippocampus correspond to the principal cell types of the CA1, $\mathrm{CA3}$, and dentate gyrus regions of the hippocampal formation. ${ }^{3,6,7}$ Typically, a place cell will fire only when the rat occupies a single restricted location in an environment (its "place field"), regardless of the rat's current behavior. Any given environment is represented by a subset of hippocampal cells, and the place fields of these cells cover the whole environment ${ }^{8}$ (although not necessarily uniformly). Place cells have been shown to be controlled by the external sensory cues in the environment, in that rotation of the cues usually causes an equal rotation of the locations of the place fields. ${ }^{6,9}$

In addition to external sensory cues, other major sources of spatial information available to the rat are internally generated (idiothetic) signals about its self-motion and position in the world. In the complete absence of external sensory information, 
rodents are able to keep track of their movements, presumably through a combination of vestibular, proprioceptive, and motor efference inputs, and generate accurate trajectories to targets. ${ }^{10} \mathrm{~A}$ major component of this "path integration" ability is an internal sense of direction, or compass, by which the rat can maintain a sense of bearing with no external sensory information. This internal direction sense is presumably mediated by another class of cells, identified in different regions of the brain, ${ }^{11-15}$ that fire strongly when the rat's head is pointed in a certain direction in allocentric space, regardless of the head's position relative to the body or the rat's position in the environment. Like place cells, these so-called head direction cells tend to be controlled by external sensory cues, in that rotation of the cues tends to cause the head direction tuning curve to rotate correspondingly. ${ }^{16}$ However, neither place cells ${ }^{17-19}$ nor head direction cells ${ }^{12,20}$ require visual input to maintain place- or direction-specific firing.

McNaughton and colleagues ${ }^{21-23}$ have developed a conceptual model of how the path integration system interacts with external sensory input to guide the rat's spatial behavior. In this model, head direction cells are considered to be driven primarily by vestibular and other idiothetic signals. If the rat is facing north, for example, and then turns clockwise 90 degrees to face east, the vestibular system detects the head's motion and, via at least one intermediate computational stage, directs the "North" head direction cells to shut off and the "East" head direction cells to begin firing. Like any inertially based compass system, though, this one is subject to cumulative error. The role of external sensory input is to reset the system when it drifts out of calibration. McNaughton and colleagues propose that this recalibration is a learned phenomenon. In a novel environment, the direction system is proposed to be completely controlled by path integration mechanisms. However, as the rat explores the environment, associations are formed between active head direction cells and those cells responding to the visual input reaching the rat at a given moment. Over time, the strengths of these associations grow, and they are eventually strong enough that the visual information is able to override the idiothetic information when the direction system drifts out of calibration. The extent to which head direction cells are controlled by visual cues or idiothetic cues in a given environment determines whether place cells will be controlled by these same cues.

If the model of McNaughton and colleagues is correct, then one would predict that visual cues would have weaker control over head direction cells and place cells if the rat is prevented from establishing strong associations between the cues and the cells. One way to do this might be to intentionally disorient the rat, such that the rat's head direction system is set at a different arbitrary bearing relative to the stable visual landmarks each time the rat enters the environment. Because there will be no consistent relationship between the rat's internal direction sense and the visual cues, no strong associations will be formed between the head direction cells and the visual neurons representing the cues. The prediction is that the visual cues will thus exert weaker control over head direction cells and place cells in disoriented rats than in rats that have always experienced a consistent, stable relationship between their internal direction sense and visual cues.

We tested this prediction by recording simultaneously head direction cells and place cells in two groups of rats as the rats moved around in a highwalled cylinder [see Ref. 24 for details]. A white cue card covering 90 degrees of the cylinder provided the only salient directional reference in the cue-controlled recording room. One group of rats was disoriented before and after each training session by being carried in a box around the perimeter of the cylinder a number of times, frequently changing direction and occasionally rotating the box gently. The other group of rats was not disoriented during training. After 1-4 weeks, the rats were surgically implanted with recording electrodes, one pair in the left hemisphere, positioned to record head direction cells in the anterior thalamic nuclei, and the other pair in the right hemisphere, positioned to record place cells in the dorsal part of area CA1. The use of the "tetrode" recording technique ${ }^{8,25}$ allowed us to record on average about three place cells with fields in the cylinder and one head direction cell in each recording session. Cells were recorded in each rat for four days, with four sessions each day. In most sessions, the cue card was in the standard location used for the initial training, but in between some sessions, it was moved to different locations, to test for visual control over the place cells and head direction 
cells. Both groups of rats were disoriented before each recording session.

The results of this experiment were clear: The visual cue had weaker control over the place cells and head direction cells in the rats that were disoriented during training than in the rats that were not disoriented. ${ }^{24} \mathrm{~A}$ typical example of place cell and head direction cell firing for a rat trained with disorientation is shown in Fig. 1. In sessions 1, 2, and 4 , the head direction cell and place cell maintained a consistent bearing relative to the visual cue (although the place field developed a weak extension in session 2). However, the head direction cell and the place cell both broke away from the visual cue control in session 3, but they maintained the same bearing relative to each other. Overall, the rats with disorientation training had a total of 28 sessions in which the place cells and head direction cells had a different bearing relative to the cue card compared to the preceding session, whereas the rats trained without disorientation had only 9 such sessions $(t=4.26$, $p=0.001$, one tailed). Importantly, the place cells and head direction cells always maintained the same bearing relative to each other, even in the sessions in which they changed bearing relative to the cue card. For a few rats, when the head direction cells rotated, about half of the place fields rotated, whereas the other half completely changed their firing properties. It appears that when these rats perceived that the cue card was in a different position relative to their own internal direction sense, the hippocampus formed a new, partially independent representation of the environment.

It should be emphasized, however, that even in the disorientation-trained rats, both cells usually were tied to the visual cue. On most recording days, there was a relatively stable, "correct" bearing of the cells relative to the cue, and the cells broke away from this bearing in about $25 \%$ of the sessions. In addition, head direction cells and place cells sometimes rotated relative to the cue card even within a particular recording session, sometimes over a brief interval ( $<1 \mathrm{~min}$ ) but often over a time span of several minutes. These rotations were usually within the first few minutes of the session, and the rotation was usually from an "incorrect" direction (based on the other recording sessions of the day) to the "correct" direction. It appears that after the disorientation procedure, the head direction cells were set at the wrong direction relative to the cue, but through some correction mechanism, the cells sometimes rotated their preferred firing direction until they locked into the correct firing direction relative to the visual cue.

These results demonstrate that hippocampal place cells and thalamic head direction cells comprise a tightly coupled system for spatial orientation, at least under the recording conditions of this experiment. An important, unresolved issue is whether the head direction system controls the place cell system, or vice-versa, or whether each system can control the other in different situations. Alternatively, it is

\section{CA1 Place Cell}
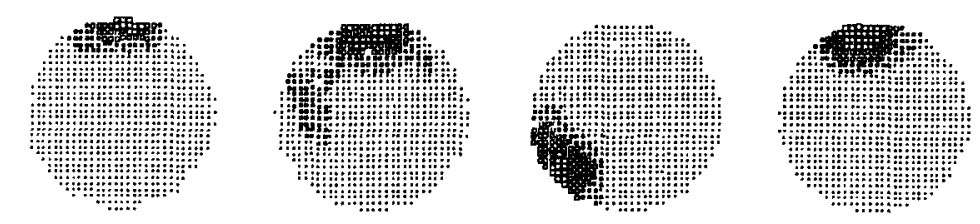

Anterior Thalamic Head Direction Cell

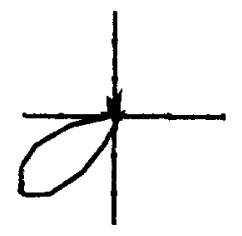

Session 1

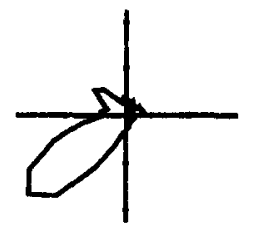

Session 2

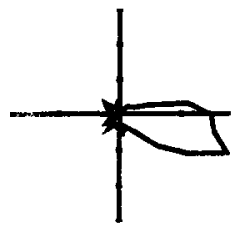

Session 3

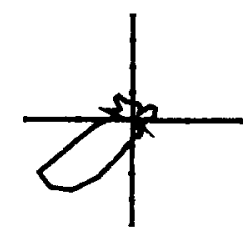

Session 4

Fig. 1. Place cell and head direction cell rotate away from the cue card in session 3 . The cue was at the 3 o'clock position in each session. 
possible that both systems are controlled by yet another input, and have no direct influence on each other. An understanding of these issues, together with information about the inputs driving these systems and behavioral analyses of the situations in which rats use different navigational cues, will help to reveal the neuronal mechanisms by which the rat generates cognitive representations of its environment and uses these representations as it learns to navigate through that environment.

A second major finding of this study is that the extent to which visual cues control the rat's spatial orientation system depends strongly on the rat's prior experience. It appears that the rat must learn that the visual cues are stable directional references in order for the cues to exert strong control. Alternatively, the cues may exert strong control by default, unless the rat learns that they are not stable directional references.

The results of the disorientation procedure are counterintuitive to many who study the behavior and neurophysiology of spatial learning and navigation. Many previous studies, including prior studies from this laboratory, have intentionally disoriented rats before trials in an attempt to enhance the control of visual cues over the rat's behavior and/or neuronal properties. $^{7,26,27}$ The logic was that when the disoriented rat experienced a continual conflict between its path integration system and the visual cues, the rat would regard the cues as the stable references and would anchor its head direction system to them, learning to ignore its unreliable path integration system. However, we suggest that just the opposite is true, that the path integration system actually has primacy over visual cues, especially in unfamiliar environments. Moreover, it is not enough for a landmark to become merely familiar for it to gain control over the path integation system. Rather, the landmark must maintain a consistent relationship with the path integration system for it to be perceived as a stable landmark and thereby eventually gain control over the head direction system.

Figure 2(a) illustrates one conceptualization of how the learned association between head direction and visual cues might develop in a rat that is not disoriented. The abscissa on each graph represents different bearings of the head direction system relative to the visual cues in an environment. The ordinate represents the state of the $n$-dimensional network determining head direction cell firing. The top graph represents the state of the network before (dashed line) and after (solid line) the first experience in the environment. In this trial, the head direction system was at a particular bearing relative to the visual cues (arbitrarily called 0 degrees), and the learned association between the visual cues and the head direction system created a small basin of attraction at that relative bearing. The middle panel illustrates the basin of attraction growing stronger as the rat experiences a consistent relationship between its head direction system and the visual cues. Finally, the bottom panel illustrates a strong basin of attraction after many experiences in the environment. If a rat with this kind of attractor was disoriented now, or if the cue card was rotated in between sessions, the rat's head direction system would be set initially at a

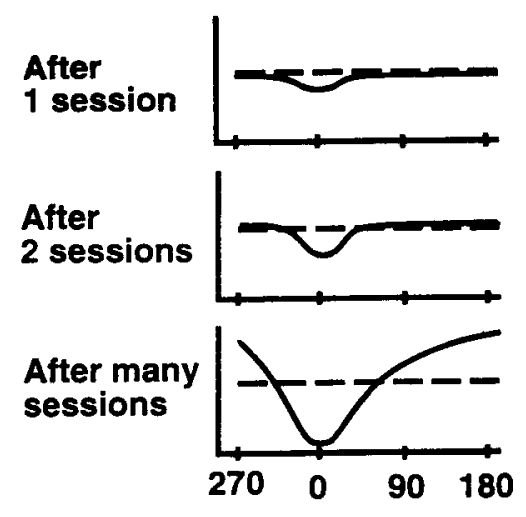

(a) No Disorientation

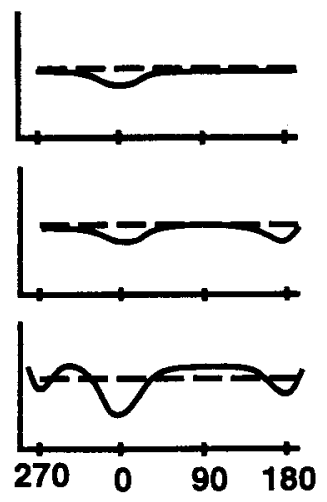

(b) Disorientation

Fig. 2. Hypothetical model of the development of visual cue control. 
different bearing relative to the cue, but the attractor dynamics would tend to pull the network into the stable state representing the learned relationship of 0 degrees, thus bringing the system in line with the visual cues.

What might be the effect of consistently disorienting the rat before each training session? Figure 2(b) illustrates the progression of learned head direction-visual cue associations in a disoriented rat. The top graph is identical to that for a rat that was not disoriented, in that a small attractor basin is established at 0 degrees (arbitrarily defined) after the first session. However, in the second session, let us assume that the head direction system starts out at a bearing 180 degrees opposite to the bearing of the first session. Since the attractor basin established in the first session is small, it does not change the firing of the head direction cells, and thus a second attractor basin is established at 180 degrees. The bottom panel illustrates what the network might look like after many experiences of disorientation. For this rat, the head direction cells will usually have the bearing associated with the 0 degrees setting, but whenever the system starts out within the basins of the other two attractors, it will instead fall toward that basin, and the visual cue will "lose" control over the cells.

We must emphasize that the above account is hypothetical at present, for the present data are insufficient to support or refute this model. However, these ideas provide a useful conceptual framework for understanding the complex interactions between path integration mechanisms and external sensory cues on the firing characteristics of head direction cells and place cells. The model's emphasis on the primacy of path integration mechanisms and the learned importance of external sensory cues may be useful in understanding such phenomena as the persistence of head direction cell and place cell firing in the $\operatorname{dark}^{12,17-20}$ as well as behavioral results demonstrating the inability of disoriented rats to use visual cues to solve certain spatial problems. ${ }^{27}$

\section{Acknowledgments}

Supported by PHS grants NS20331 and NS09052, ONR grant N0014-90-J-1869, by the Human Frontiers Science Program, and by the McDonell-Pew Program.

\section{References}

1. J. O'Keefe and L. Nadel 1978, The Hippocampus as a Cognitive Map (Clarendon Press, Oxford).

2. L. E. Jarrard 1993, "On the role of the hippocampus in learning and memory in the rat," Behav. Neur. Biol. 60, 9-26.

3. J. O'Keefe and J. Dostrovsky 1971, "The hippocampus as a spatial map: Preliminary evidence from unit activity in the freely-moving rat," Brain Res. 34, 171-175.

4. L. R. Squire 1987, Memory and Brain (Oxford University Press, New York).

5. H. Eichenbaum, T. Otto and N. J. Cohen 1994, "Two functional components of the hippocampal memory system," Behav. Brain Sci. 17, 449-517.

6. R. U. Muller and J. L. Kubie 1987, "The effects of changes in the environment on the spatial firing of hippocampal complex-spike cells," J. Neurosci. 77, 1951-1968.

7. M. W. Jung and B. L. McNaughton 1993, "Spatial selectivity of unit activity in the hippocampal granular layer," Hippocampus 3, 165-182.

8. M. A. Wilson and B. L. MeNaughton 1993, "Dynamics of the hippocampal ensemble code for space," Science 261, 1055-1058.

9. J. O'Keefe and A. Speakman 1987, "Single unit activity in the rat hippocampus during a spatial memory task," Exp. Brain Res. 68, 1-27.

10. M. L. Mittelstaedt and H. Mittelstaedt 1980, "Homing by path integration in a mammal," Naturwissenschaften 67, 566-567.

11. J. S. Taube, R. U. Muller and J. B. Ranck.Jr. 1990, "Head direction cells recorded from the postsubiculum in freely moving rats. I. Description and quantitative analysis," J. Neurosci. 10, 420-435.

12. S. J. Y. Mizumori and J. D. Williams 1993, "Directionally selective mnemonic properties of neurons in the lateral dorsal nucleus of the thalamus of rats," J. Neurosci. 13, 4015-4028.

13. S. I. Wiener 1993, "Spatial and behavioral correlates of striatal neurons in rats performing a self-initiated navigation task," J. Neurosci. 13, 3802-3817.

14. L. L. Chen, L.-H. Lin, E. J. Green, C. A. Barnes and B. L. McNaughton 1994, "Head direction cells in the rat posterior cortex: I. Anatomical distribution and behavioral modulation," Exp. Brain Res. 101, 8-23.

15. J. S. Taube 1995, "Head direction cells recorded in the anterior thalamic nuclei of freely moving rats," J. Neurosci. 15, 70-86.

16. J. S. Taube, R. U. Muller and J. B. Ranck, Jr. 1990, "Head direction cells recorded from the postsubiculum in freely moving rats. II. Effects of environmental manipulations," J. Neurosci. 10, 436-447.

17. B. L. McNaughton, B. Leonard and L. Chen 1989, "Cortical-hippocampal interactions and cognitive mapping: A hypothesis based on reintegration of the 
parietal and inferotemporal pathways for visual processing," Psychobiology 17, 236-246.

18. G. J. Quirk, R. U. Muller and J. L. Kubie 1990, "The firing of hippocampal place cells in the dark depends on the rat's recent experience," J. Neurosci. 10, 2008-2017.

19. E. J. Markus, C. A. Barnes, B. L. McNaughton, V. L. Gladden and W. E. Skaggs 1994, "Spatial information content and reliability of hippocampal CA1 neurons: Effects of visual input," Hippocampus 4, 410-421.

20. L. L. Chen, L.-H. Lin, C. A. Barnes and B. L. McNaughton 1994, "Head direction cells in the rat posterior cortex: II. Contributions of visual and ideothetic information to the directional firing," Exp. Brain Res. 101, 24-34.

21. B. L. McNaughton, L. L. Chen and E. J. Markus 1991, "'Dead reckoning,' landmark learning, and the sense of direction: A neurophysiological and computational hypothesis," J. Cognit. Neurosci. 3, 190-202.

22. B. L. McNaughton, J. J. Knierim and M. A. Wilson 1995, "Vector encoding and the vestibular foundations of spatial cognition: Neurophysiological and computational mechanisms," in The Cognitive Neurosciences, ed. M. Gazzaniga (MIT Press, Boston), pp. 585-595.
23. W. E. Skaggs, J. J. Knierim, H. S. Kudrimoti and B. L. McNaughton 1995, "A model of the neural basis of the rat's sense of direction," in Adv. in Neural Info. Processing Systems 7, eds. D. S. Touretzky, G. Tesauro and T. K. Leen (MIT Press, Cambridge), pp. 173-180.

24. J. J. Knierim, H. S. Kudrimoti and B. L. McNaughton 1995, "Place cells, head direction cells, and the learning of landmark stability," $J$. Neurosci. 15, 1648-1659.

25. M. L. Recce and J. O'Keefe 1989, "The tetrode: An improved technique for multi-unit extracellular recording," Soc. Neurosci. Abstr. 15, 1250.

26. R. Biegler and R. G. M. Morris 1993, "Landmark stability is a prerequisite for spatial but not discrimination learning," Nature 361, 631-633.

27. K. Cheng 1986, "A purely geometric module in the rat's spatial representation," Cognition, 23, 149-178. 